nach Asien und in die USA und kamen erst 1947 wieder in Schweden an. Als Ruth Jacoby, dann schon in Berlin tätig, in der Literaturhandlung in der Joachimstaler Straße den Namen ihres Vaters im Begleitband zur Ausstellung „Anwalt ohne Recht“ entdeckte, ergaben sich daraus der Kontakt zur Berliner Rechtsanwaltskammer bzw. deren damaliger Präsidentin Dr. Margarete von Galen und die weiteren Verbindungen zu den Berliner Jurist(inn)en. Darunter auch Prof. (Asoc.) Dr.
Jutta Glock, Vorsitzende des Landesverbands Berlin im djb. Auf deren Einladung stellte Ruth Jacoby in einer Rede zum Sommerempfang des djb-Landesverbands 2009 umfassend dar, dass und warum Schweden mit der Gleichstellung von Frauen und Männer Deutschland 30 Jahre voraus ist. Der Global Gender Gap Report des World Economic Forum, der die Gleichstellung der Geschlechter analysiert, setzte Schweden mehrfach auf den ersten Platz.

\title{
Die Abteilung Arbeits- und Sozialrecht beim 68. Deutschen Juristentag in Berlin
}

\section{Gisela Ludewig \\ Mitglied der Kommission Arbeits-, Gleichstellungs- und Wirt- schaftsrecht des djb; Rechtsanwältin, Berlin}

Der Deutsche Juristentag, der in Berlin sein 150. Jubiläum gefeiert hat, fasste am 23. September 2010 einige interessante Beschlüsse zum Arbeits- und Sozialrecht.

Das Thema der von Prof. Dr. Gregor Thüsing, LL.M., Bonn ${ }^{1}$ geleiteten Abteilung lautete: „Abschied vom Normalarbeitsverhältnis? - Welche arbeits- und sozialrechtlichen Regelungen empfehlen sich im Hinblick auf die Zunahme neuer Beschäftigungsformen und die wachsende Diskontinuität von Erwerbsbiografien?“

Das Gutachten war erstellt worden von Prof. Dr. Raimund Waltermann, Bonn. Die Referenten waren Mitglied des Vorstands der Bundesagentur für Arbeit Heinrich Alt, Nürnberg, Prof. Dr. Wolfhard Kohte, Halle und Prof. Dr. Christian Rolfs, Köln.

Das Normalarbeitsverhältnis ist gekennzeichnet durch eine unbefristete Vollzeitbeschäftigung oder Teilzeittätigkeit mit mindestens der Hälfte der üblichen Arbeitszeit beim Vertragsarbeitgeber bei angemessener Vergütung. Es ist in die Systeme der sozialen Sicherheit integriert und somit geeignet, den Lebensunterhalt einer Person einschließlich der sozialen Absicherung auf Dauer zu gewährleisten.

Seit Beginn der Neunzigerjahre führt nicht nur eine Veränderung der tatsächlichen Verhältnisse durch äußere Umstände, insbesondere durch die Situation am Arbeitsmarkt, zu einer Abnahme der den Lebensstandard sichernden Normalarbeitsverhältnisse und zum Aufbau der sogenannten „atypischen Beschäftigungsformen " und ertragsschwachen Normalarbeitsverhältnisse. Auch die Veränderung der Rechtslage hat hierzu beigetragen. Gemeint sind Leiharbeit, befristete Beschäftigung und Teilzeitbeschäftigung, vor allem in Form der geringfügig entlohnten Beschäftigung, aber auch Selbständige ohne Mitarbeiter ${ }^{2}$ und Beschäftigung im Niedriglohnsektor.

Die arbeits- und sozialrechtliche Abteilung des 68. djt setzte es sich zur Aufgabe, die aus dem tatsächlichen Befund - der zum einem im Gutachten von Prof. Dr. Raimund Waltermann dargestellt war, zum andern durch den Vortrag des Vorstandsmitglieds der Bundesagentur für Arbeit Heinrich Alt untermauert wurde - erwachsenden rechtlichen Folgen zu bewerten und die Frage zu beantworten, ob diese Entwicklungen zu einer Abkehr vom Normalarbeitsverhältnis führen, die man durch Gesetzgebung fördern, verhindern oder gestalten müsste. Bemerkenswert war, dass zum Befund die Feststellung gehörte, dass die „atypischen Beschäftigungsformen“ sich als kaum geeignet erwiesen haben, um den Übergang in ein Normalarbeitsverhältnis zu ebnen („Brückenfunktion“). Statt von einer Brücke könne man allenfalls von einem „Steg“ sprechen, so Alt.

Die Fragestellung der arbeits- und sozialrechtlichen Abteilung ist nicht nur für die Entwicklung der Arbeitsbeziehungen von Bedeutung. Weil in Deutschland die sozialversicherungsrechtliche Absicherung an die Beschäftigung in abhängiger Arbeit anknüpft, ist auch die Zukunft der sozialen Vorsorge betroffen.

Vor allem die gesetzliche Rentenversicherung vermittelt ihr Sicherungsniveau in Anknüpfung an die Erwerbsbiografien, die in einem Normalarbeitsverhältnis zurückgelegt werden. Auch der Krankenversicherungsschutz setzt ein solides Beitragsaufkommen voraus. Die Einnahmebasis der Krankenkassen wird durch die „atypischen Beschäftigungsformen“ geschmälert, insbesondere durch die abgabenprivilegierten $\mathrm{Mi}$ nijobs.

Der djb unterstützte nachdrücklich den Prüfauftrag des Gutachters Prof. Dr. Raimund Waltermann, die Abgabenprivilegierung der Minijobs abzuschaffen, da diese Minijobs der gleichstellungspolitisch notwendigen eigenständigen Existenzsicherung von Frauen entgegenwirken, sich als berufliche Sackgasse und ein Hindernis auf dem Weg zur Überwindung des „equal pay gap“ erwiesen haben, wie der djb in seiner Pressemitteilung vom 20. September 2010 betonte.

Gutachten und Thesen der Referenten wurden am Mittwoch und Donnerstag intensiv diskutiert. Die Teilnehmer-

\footnotetext{
1 Stellvertreter: Vors. Richter am BSG Prof. Dr. Peter Udsching, Kassel/ Göttingen.

2 Sog. „Kleine Selbständigkeit“.
} 
(innen) waren sich einig, dass das sogenannte Normalarbeitsverhältnis ein Leitbild der Rechtsbeziehungen im Arbeitsrecht sei, das der Gesetzgeber durch intelligente Lösungen stützen solle. ${ }^{3}$

Insgesamt fasste die Abteilung 36 Beschlüsse, zu den zuvor durchaus kontrovers behandelten Themen unter anderem folgende:

Das Vorhaben des Koalitionsvertrages, die geringfügige Beschäftigung über die Einkommensgrenze von 400 Euro monatlich auszuweiten, ist nicht sachgerecht. ${ }^{4}$

Der Gesetzgeber sollte die abgabenrechtliche Privilegierung der geringfügigen Beschäftigung aufgeben. Deren beschäftigungspolitische Ziele und deren Vorteile für Arbeitgeber(innen) und Arbeitnehmer(innen) werden durch beträchtliche Nachteile für die Gesellschaft erkauft, zu denen insbesondere auch eine Verlagerung von Gegenwartsproblemen in das Steueraufkommen der Zukunft gehört. Um eine behutsame Überführung der bestehenden geringfügigen Beschäftigungsverhältnisse in regulär besteuerte und verbeitragte Arbeitsverhältnisse zu ermöglichen, könnte es sich empfeblen, in einem ersten Schritt allein die sozialversicherungsrechtliche Sonderbehandlung aufzuheben, die steuerrechtliche aber zunächst beizubehalten. Damit würde sich der Kostensprung in Grenzen halten. Später könnte dann der Pauschalsteuersatz von derzeit zwei Prozent ${ }^{5}$ kontinuierlich angehoben werden und allmählich in den individuellen Steuersatz der (des) Arbeitnehmerin (Arbeitnehmers) einmünden. Mit der Aufgabe der sozialversicherungsrechtlichen Privilegierung geringfügiger Beschäftigungsverhältnisse ist die Abschaffung der seit 2003 bestehenden sogenannte „Gleitzone "6 sachlogisch verbunden.

Im Befristungsrecht soll $\mathbb{} 14$ Abs. 3 TzBfG gestrichen werden, wonach derzeit, anknüpfend an ein Alter von 52 Jahren, befristete Arbeitsverhältnisse ohne Vorliegen eines sachlichen Grundes bis zur Dauer von fünf Jahren zulässig sind.

Der Antrag, das Verbot der Vorbeschäftigung in $\mathbb{S} 14 \mathrm{Ab}$ satz 2 Satz $2 \mathrm{TzBfG}^{7}$ durch eine Mindestunterbrechungsdauer von einem Jahr zu ersetzen, ist abgelehnt worden.

Eine Aufweichung von $\mathbb{} 12 \mathrm{TzBfG}^{8}$ zugunsten der Dispositionsbefugnis der (des) Arbeitgeberin (Arbeitgebers) ist abgelehnt worden.

Zur Teilzeitbeschäftigung wurde ein Antrag angenommen, wonach diese künftig auch für einen befristeten Zeitraum beansprucht werden kann. Derzeit ist der Rechtsanspruch gemäß \ 8 TzBfG nur auf eine dauerhafte Verringerung der Arbeitszeit gerichtet, der Anspruch der (des) teilzeitbeschäftigten Arbeitnehmerin (Arbeitnehmers) auf Verlängerung der Arbeitszeit nach $\mathbb{9}$ TzBfG ist in der Praxis schwer durchsetzbar.

Zustimmung erhielt auch der Antrag, dass ein Verhandlungsanspruch hinsichtlich der Gestaltungsmöglichkeiten über das Arbeitsarrangement nach Rückkehr aus der Elternzeit entsprechend der RL 210/18/EU in $\$ 15$ BEEG umgesetzt werden soll.

Zur Leiharbeit wurde beschlossen, dass die Ausnahmeregelung des $\mathbb{9}$ Nr. 2 AÜG 9 aufzuheben ist.
Angenommen wurde auch ein Antrag, wonach die Möglichkeit aufzugeben ist, dass die Leiharbeitsbranche durch Tarifverträge mit ungünstigeren Arbeitsbedingungen vom Gleichbehandlungsgrundsatz abweicht.

Last but not least beschloss die Abteilung Arbeits- und Sozialrecht, dem Gesetzgeber die Einführung eines einheitlichen allgemeinen Mindestlohns zu empfehlen:

Bei der Frage, ob nicht ein flächendeckender allgemeiner Mindestlobn eingeführt werden sollte, ist aus rechtswissenschaftlicher Perspektive der Zusammenhang zwischen sozialrechtlichen Transferleistungen ${ }^{10}$ und dem privatautonom vereinbarten Arbeitsentgelt in den Blick zu nebmen. Dieser Zusammenhang von Sozialrecht und Privatrecht, der die Grundsicherung und andere Transferleistungen zu einem Kombilohnmodell macht, beeinflusst die Marktsituation am Arbeitsmarkt im Niedriglohnbereich. Diesem Befund kann durch einen einheitlichen allgemeinen Mindestlohn als fixierter Untergrenze entgegengewirkt werden. Für die konkrete Ausgestaltung des Mindestlohns liefern unsere Nachbarstaaten erprobte Handlungsmuster. Ein geeignetes Vorbild ist das britische Kommissionsmodell, das mit einer intensiven Beteiligung der Tarifvertragsparteien und einer sorgfältigen zeitnahen Untersuchung des Arbeitsmarkts verbunden ist und das auch von der neuen britischen Regierung fortgesetzt wird.

Unter den hier nicht im Einzelnen angesprochenen Beschlüssen finden sich auch einige zur Stärkung kontinuierlicher Erwerbsbiografien und zur sozialversicherungsrechtlichen Absicherung bei der sogenannten „Kleinen Selbständigkeit".

3305 Ja-Stimmen, o Gegenstimmen, 1 Enthaltung.

4304 Ja-Stimmen, 4 Gegenstimmen, 8 Enthaltungen.

$5 \S 40 a$ Abs. 2 EStG.

$6 \S 20$ Abs. 2 SGB IV.

7 Unzulässigkeit einer Befristung ohne sachlichen Grund, wenn mit demselben Arbeitgeber jemals zuvor ein Arbeitsverhältnis bestanden hat.

8 Arbeit auf Abruf.

9 Absenkung des Eingangsentgelts.

10 Namentlich Grundsicherung nach dem SGB II - „Hartz IV“, Familienzuschlag gemäß $\S 6$ a BKGG, Wohngeld. 